\title{
Effects of soil spatial variability on axisymmetric versus plane strain analyses of ground improvement by prefabricated vertical drains
}

\author{
Md. Wasiul Bari† \\ $\mathrm{PhD}$ Candidate, Department of Civil Engineering, \\ Curtin University, WA 6845, Australia \\ Phone: +61-8-9266 1783 \\ E-mail: mdwasiul.bari@postgrad.curtin.edu.au
}

\author{
Mohamed A. Shahin \\ Senior Lecturer, Department of Civil Engineering, \\ Curtin University of Technology, WA 6845, Australia \\ Phone: +61-8-9266 1822; Fax: +61-8-9266 2681 \\ E-mail: M.Shahin@curtin.edu.au
}

\author{
Hamid R. Nikraz \\ Professor, Department of Civil Engineering, \\ Curtin University of Technology, WA 6845, Australia \\ Phone: +61-8-9266 7573; Fax: +61-8-9266 2681 \\ E-mail: $\underline{\text { H.Nikraz@curtin.edu.au }}$
}

$\uparrow$ Corresponding author

Submitted to: International Journal of Geotechnical Engineering 


\title{
Effects of soil spatial variability on axisymmetric versus plane strain analyses of ground improvement by prefabricated vertical drains
}

\author{
Md. Wasiul Bari, Mohamed A. Shahin and Hamid R. Nikraz
}

\begin{abstract}
Although soil consolidation around an individual drain is more appropriately analyzed as an axisymmetric problem, most previous numerical studies regarding soil consolidation via prefabricated vertical drains were conducted on the basis of plane strain conditions so as to achieve run-time efficiency. Under deterministic conditions, it was found in the literature that the matching theories give satisfactory equivalence between plane strain and axisymmetric analyses. Nevertheless, the matching theories have never been examined for the more realistic stochastic soil consolidation approaches and this study aims to investigate such an issue. In this paper, a stochastic approach using the Monte Carlo technique is utilized considering soil permeability as the most significant random field and the equivalence between the axisymmetric and plane strain analyses are examined.
\end{abstract}

KEYWORDS: Finite elements, soil variability, consolidation, vertical drains.

\section{INTRODUCTION}

Prefabricated vertical drains (PVDs) in combination with pre-loading are a popular technique that is used to enhance the bearing capacity of soft soil by accelerating soil 
consolidation. In traditional PVD design approaches, the consolidating soil deposits are assumed to be homogeneous with constant values of soil properties across the soil mass. Consequently, for erratic soils, most available design methods (e.g., Barron 1948, Hansbo 1981, Hird et al. 1992) failed to provide satisfactory predictions of the soil consolidation rates due to the substantial variation of soil properties in space (e.g., compressibility and permeability coefficients in the vertical and horizontal directions). This inherent spatial variation of soil properties indicates that soil consolidation via PVDs is highly compliant to a statistical interpretation. Although the stochastic nature of soil properties and the importance of the reliability analyses are recently recognized in many geotechnical engineering applications, little research has been done on soil consolidation by PVDs considering soil spatial variability. In recent years, a few studies that consider soil spatial variability have been conducted for soil consolidation due to vertical drainage only (i.e., without PVDs) in 1D and 2D geometries (e.g., Huang et al. 2010, Hwang and Witczak 1984), and the only study found in the literature for soil spatial variability in soil consolidation via PVDs has been carried out by the authors (i.e., Bari et al. 2010)

The consolidation of soil around an individual drain is more appropriately analysed as an axisymmetric problem. However, most previous numerical studies regarding soil consolidation via PVDs were conducted on the basis of plane strain assumption so as to achieve computational efficiency. Analysing an axisymmetric problem using plane strain conditions requires the equivalence between the axisymmetric and plane strain analyses. By employing the established matching theories (e.g., Hird et al. 1992, Indraratna and Redana 1997), it was found in the literature that under deterministic conditions, satisfactory equivalence between the plane strain and axisymmetric analyses 
is achievable. However, to the authors' best knowledge, the matching procedures have not been tested yet for the more realistic stochastic soil consolidation approaches. Accordingly, in the current study, stochastic analyses are performed for both axisymmetric and equivalent plane strain cases, considering soil permeability as the random field and the comparison between the axisymmetric and plane strain analyses are critically examined and discussed.

\section{OVERVIEW OF ANALYTICAL SOLUTION OF SOIL CONSOLIDATION BY PVDs AND MATCHING PROCEDURE FOR EQUIVALENT PLANE STRAIN ANALYSIS}

Permeability in the horizontal direction of most geologic deposits is often much higher than that of the vertical direction (Hansbo 1981). Therefore, the key feature of soil stabilization by PVDs is to accelerate the primary consolidation of soft soils by providing fast drainage in the horizontal direction while applying a pre-consolidation loading (usually in the form of an embankment or vacuum pressure). The installation of PVDs into the ground provides much shorter radial drainage path of less water flow resistance in the compressible soil mass, thus, significantly reduces the consolidation time. In such case, the overall soil consolidation results from the vertical drainage of natural subsoil and the horizontal (radial) drainage due to existence of the PVDs. However, in practical sense, consolidation due to vertical drainage is much less than that of the horizontal drainage and thus can be neglected. Consequently, in the current study, only soil consolidation due to the horizontal drainage is analysed. The axisymmetric analytical solution of soil consolidation due to horizontal drainage is 
presented by Hansbo (1981) and has gained a wide acceptance in practical application. The solution accounts for the effects of both smear and well resistance, and estimates the degree of consolidation, $U_{h}(t)$, on a horizontal plane at depth $z$ and time $t$ as follows:

$U_{h}(t)=1-\exp \left(-\frac{2 k_{h} t}{m_{v} \gamma_{w} R_{e}^{2} \alpha}\right)$

In which:

$$
\alpha=\ln \left(\frac{n}{s}\right)+\left(\frac{k_{h}}{k_{s}}\right) \ln (s)-0.75+\pi z(2 L-z) \frac{k_{h}}{q_{w}}
$$

where: $k_{h}$ is the coefficient of permeability in the horizontal direction; $m_{v}$ is the coefficient of volume compressibility; $\gamma_{w}$ is the unit weight of water; $R_{e}$ is the radius of the equivalent soil cylinder with impermeable perimeter or the radius of zone of influence; $t$ is the consolidation time; $\alpha$ is a group parameter representing the smear effects and geometry of the PVD system; $n=R_{e} / r_{w}$ is the drain spacing ratio ( $r_{w}$ is the equivalent radius of the drain); $s=r_{s} / r_{w}$ is the smear ratio ( $r_{s}$ is the radius of the smear zone); $k_{s}$ is the horizontal permeability of the smear zone; $L$ is the maximum vertical drainage distance; $z$ is the depth from the top of the consolidating layer; and $q_{w}$ is the vertical discharge capacity of the drains. All parameters in Eqs. (1) and (2) are illustrated in Figure 1.

The conversion techniques of axisymmetric solution to an equivalent plane strain model are demonstrated by several researchers (e.g., Hird et al. 1992, Indraratna and Redana 
1997). This can be done by: (i) geometric matching in which the spacing of the drains is matched while the permeability is reserved the same; (ii) permeability matching in which the permeability coefficients are matched while the drain spacing is reserved the same; and (iii) combination of permeability and geometric matching approach in which the plane strain permeability is computed for convenient drain spacing (Indraratna et al. 2003). Out of the above three matching theories, the permeability matching technique is considered for the stochastic analyses carried out in the current study. It should be noted that the installation process of PVDs into the ground results in a zone of reduced permeability surrounding the drain periphery known as the smear zone. The reduced permeability in the smear zone is also spatially variable (Bo et al. 2003) and may affect the process of soil consolidation. The well resistance is another factor that may also retard the rate of consolidation and is originated mainly from the drain clogging by infiltration of fine soil grains and drain folding due to the high lateral earth pressure. However, the effects of smear and well resistance are not considered in the stochastic study as the matching procedure is deemed to be examined in its simplest case, and more complicated disparities on this subject is left for future refinement. Without considering the smear and well resistance effects, the permeability in the plane strain, $k_{p l}$, can be estimated from the permeability of the axisymmetric, $k_{a}$, using the following transformation function (Hird et al. 1992):

$k_{p l}=\frac{0.67}{[\ln (n)-0.75]} k_{a}$

For the plane strain analysis to be applied so as to achieve an equivalent degree of consolidation to that of the axisymmetric analysis at any consolidation time, $k_{p l}$ should 
be used in Eqs. (1) and (2). In the following section, the detailed procedure of developing stochastic finite element analyses for soil consolidation by PVDs under axisymmetric and equivalent plane stain conditions that take into account spatial variability of soil permeability are described and discussed.

\section{STOCHASTIC MODELLING OF SOIL CONSOLIDATION BY PVDS}

This paper presents stochastic analyses of soil consolidation by PVDs considering associated uncertainty due to spatial variation of soil permeability. In a Monte Carlo framework, the approach merges the random field theory (Vanmarcke 1984) for generating random permeability fields and the finite element method. The steps of the stochastic approach are as follows:

1. Characterize and generate the soil permeability random field;

2. Incorporate the generated values of soil permeability into a finite element modelling of soil consolidation by PVDs; and

3. Repeat Steps 1 and 2 many times using the Monte Carlo technique so that a series of consolidation responses can be obtained from which the consolidation behaviour in the form of probability distribution functions can be investigated.

The above steps, as well as the analytical and numerical procedures, are described in detail below. 


\subsection{Characterization and Generation of a Random Permeability Field}

Although spatial variability of several soil properties can affect the consolidation of heterogeneous soils, the coefficient of volume compressibility and coefficient of soil permeability play the most crucial roles as indicated by several researchers (e.g., Lee et al. 1992, Pyrah 1996). However, in the current study, only the permeability is considered to be spatially random and coefficient of volume compressibility is assumed to be constant because of the fact that soil permeability has the highest range of variation amongst all other soil parameters (Bo et al. 2003) and its coefficient of variation is usually much higher than that of volume compressibility (Huang et al. 2010). As mentioned earlier, only soil consolidation due to horizontal drainage is considered in the current study and for convenience, the coefficient of horizontal permeability, $k_{h}$, will be simply denoted as $k$. Since $k$ is a non-negative parameter that was suggested in the literature to follow a lognormal distribution based on field tests data (Hoeksema and Kitanidis 1985), it is also assumed in the current study to follow a lognormal distribution. The statistical characteristics of $k$ are described by its mean, $\mu_{k}$, and standard deviation, $\sigma_{k}$. Although soil properties vary randomly in space, such variation is gradual and thus spatially correlated. In order to take into account the spatial correlation between the random permeability values, a third statistical parameter is needed, i.e., the spatial correlation length or scale of fluctuation, $\theta_{\ln k}$. The correlation length describes the limit of the spatial continuity of spatial correlation and can simply be expressed as the distance over which the soil properties show considerable correlation between two spatial points. Therefore, a large value of $\theta_{\ln k}$ indicates a smoothly varying field, whereas a small value of $\theta_{\ln k}$ implies an erratic field. 
To simulate the random permeability field of $k$, first a correlated local average normal random field $g_{\operatorname{lnk}}(i)$ over the domain of the $i$ th element is generated from a sequence of standard normal random field $g_{\ln k}$ having zero mean, unit variance and spatial correlation length $\theta_{\ln k}$ using the local average subdivision (LAS) method (Fenton and Vanmarcke 1990). LAS is used as it simplifies the task of generating finite elements from the random field and has the ability to increase the resolution of certain regions in the finite element modelling of random fields (Paice et al. 1994). The log-normally distributed permeability field $k_{i}$ of the $i$ th element is then obtained through the following transformation function (Fenton and Griffiths 2008):

$k_{i}=\exp \left\{\mu_{\ln k}+\sigma_{\ln k} g_{\ln k}(i)\right\}$

where: $\mu_{\ln k}$ and $\sigma_{\ln k}$ are, respectively, the mean the standard deviation of the logarithm of $k$ obtained from the specified permeability $\mu_{k}$ and $\sigma_{k}$ using the following transformation functions (Fenton and Griffiths 2008):

$$
\begin{aligned}
& \sigma_{\ln k}=\sqrt{\ln \left(1+\frac{\sigma_{k}^{2}}{\mu_{k}^{2}}\right)} \\
& \mu_{\ln k}=\ln \mu_{k}-\frac{1}{2} \sigma_{\ln k}^{2}
\end{aligned}
$$

The spatial correlation of $k$ is estimated mathematically using an exponentially decaying (Markovian) spatial correlation function, as follows (Fenton and Griffiths 2008): 
$\rho_{\ln k}=\exp \left(-\frac{2|\tau|}{\theta_{\ln k}}\right)$

where: $|\tau|$ is the absolute distance between two points in the soil domain. It is worthy to note that the spatial correlation length is estimated with respect to the underlying normally distributed permeability field $(\ln k)$ and operates between the values of $\ln k$. It should be also noted that the random permeability field generated by employing the LAS technique is two dimensional in which the spatial correlation structure of soil permeability is assumed to be statistically isotropic, i.e., the scales of fluctuation in the vertical and horizontal directions are assumed to be the same (i.e., $\theta_{h_{\ln k}}=\theta_{v_{\ln k}}=\theta_{\ln k}$ ). Although, in natural soil deposits, the scale of fluctuation cannot be expected to be isotropic due to the layering and stratification processes, the isotropic correlation length is employed herein for providing a common non-site specific study, which is believed to be logical. It has to be noted that, since the random permeability field is generated by employing a 2D model, the scale of fluctuation in the circumferential direction of the axisymmetric solution will be infinite and thus the soil properties in this direction remain constant. Likewise, the scale of fluctuation in the out-of-plane direction of the plane strain solution will also be infinite and thus the soil properties in this direction remain constant. Despite the above limitation, it is believed that the two-dimensional model will still yield valuable insights. 


\subsection{Finite Elements Modelling Incorporating Soil Spatial Variability}

With the random permeability field has been generated in Section 3.1 by assigning the random field parameters $\mu_{k}, \sigma_{k}$ and $\theta_{\ln k}$ into the LAS method, the spatial variability of soil permeability can then be employed as input in a computational scheme for soil stabilisation by PVDs. In this study, all computational analyses of soil consolidation via PVDs are performed using the finite element computer code AFENA (Carter and Balaam 1995), in which the consolidation problem is treated as a coupled transient problem governed by the Biot's consolidation theory (Biot 1941). In the Biot's theory, isotropic consolidation of saturated media is represented through coupled solid-fluid interaction equations, formulated by the condition of equilibrium and continuity. The finite element discretization and solution by the Galerkin method transform the equilibrium and continuity equations into a set of element matrix equations, which can be resolved by applying the fully implicit integration technique, and these matrix equations can be expressed as follows:

$$
\left[k_{m}\right]\{\delta\}+[c]\{u\}=\{f\}
$$

$$
[c]^{T}\left\{\frac{d \delta}{d t}\right\}-[\kappa]\{u\}=\{0\}
$$

where: $\left[k_{m}\right]$ and $[\kappa]$ are the material stiffness and permeability matrices, respectively; $[c]$ is the coupling matrix; and $\{u\},\{f\}$ and $\{\delta\}$ are, respectively, the excess pore pressure, total applied load and displacement. 
Before using AFENA in the stochastic analyses, the the ability of the code in simulating soil consolidation by PVDs is tested under deterministic axisymmetric conditions against an example solved by Hird et al. (1992) using the finite element software CRISP. In addition, AFENA is further verified for the same example against the analytical solution of Hansbo (1981) using Eq. (1) and the results are shown in Figure 2, which illustrate an excellent agreement. It should be noted that the term $T_{h}$ in the horizontal axis of Figure 2 is known as the time factor for radial (horizontal) drainage and is equal to $k_{h} t / 4 m_{v} \gamma_{w} R_{e}^{2}$.

Since a single-drain analysis is often enough to investigate the soil consolidation behaviour, the validity of the permeability matching theory is examined stochastically using a unit cell of soil around a single drain without considering smear and well resistance effects. The consolidation problem considered in the current study implies a geometry $L=1.0 \mathrm{~m}, R_{e}=B=0.85 \mathrm{~m}(B$ is the half width of the unit cell for the plane strain analysis) and $r_{w}=b_{w}=0.05 \mathrm{~m}\left(b_{w}\right.$ is the half width of the drain) and the applied boundary conditions for this problem are shown in Figure 1. The soil mass is modelled as a linear elastic material with spatially variable coefficient of permeability, $k$, constant modulus of elasticity $E^{\prime}=5 \mathrm{MPa}$ and Poisson's ratio $v^{\prime}=0.25$. The mean permeability, $\mu_{k}$, for the axisymmetric is selected to be equal to $5 \times 10^{-10} \mathrm{~m} / \mathrm{sec}$. It should be emphasized that, in order to establish the necessary matching between the axisymmetric and plane strain analyses, the mean permeability of the plane strain is computed by making use of the mean axisymmetric permeability of $5 \times 10^{-10} \mathrm{~m} / \mathrm{sec}$ into Eq. (3). Thus, the resulting mean permeability of plane strain would be equal to $1.6 \times 10^{-10} \mathrm{~m} / \mathrm{sec}$. Since the mean permeability for equivalent plane strain case is different from that of the axisymmetric, for the purpose of comparison, $\sigma_{k}$ is expressed in terms of the 
dimensionless coefficient of variation, $\omega$, where: $\omega=\sigma_{k} / \mu_{k}$ and for convenience, $\theta_{\ln k}$ is simply referred to as $\Theta$ throughout the rest of the paper. In this research, the equivalence between the axisymmetric and equivalent plane strain analyses using the permeability matching theory is examined over the range of the following $\omega$ and $\Theta$ :

- $\omega(\%)=25,50,100,200$ and 400

- $\Theta=0.125,0.25,0.5,1.0,2.0$ and 4.0

Although, the accuracy of the finite element solutions increases with the increase of the number of elements in the mesh, a trade-off between accuracy and run-time efficiency is necessary. In this work, the finite-element mesh is discretized into $16 \times 20$ square elements each contains eight bi-quadratic displacement nodes and four bilinear pore pressure nodes. It has to be noted that although in the plane strain analysis, there is no axis of symmetry for heterogeneous soil, only one half of the unit cell is modelled, as a comparison carried out considering full and half unit cell showed no significant difference because of the fact that the number of elements in the mesh and the number of Monte Carlo simulations considered in this study are sufficient to produce reasonably reproducible result.

\subsection{Repetition of Process Based on the Monte Carlo Technique}

Following the procedures of the Monte Carlo technique, the process of generating a random permeability field (Section 3.1) and the subsequent finite element analysis (Section 3.2) is repeated many times until certain convergence is achieved. It was found that 1000 Monte Carlo simulations yield reasonably stable statistics of the degree of consolidation. Each new generation of random field over the finite element mesh and 
the subsequent finite element analysis is termed a "realization". Figure 3 shows the discretized mesh and the corresponding soil domain represented by a grey scale of a typical permeability field realization in which the dark and light regions indicate, respectively, "smaller" and "larger" values of permeability. In each realization, the same $\mu_{k}, \sigma_{k}$ and $\Theta$ are used; however, the spatial distribution of soil permeability varied from one realization to the next. Using each combination of the statistical parameters $\omega$ and $\Theta$ specified in Section 3.2 above, a series of 30 stochastic simulations are carried out for the axisymmetric solution and another 30 tests are performed for the equivalent plane strain conditions. The obtained outputs from the suite of 1000 realizations of the Monte Carlo technique are collated and statistically analysed to estimate the mean and standard deviation of the degree of consolidation. At any stage of consolidation of time $t$, the mean (over the 1000 realizations of the Monte Carlo simulations) of the degree of consolidation, $\mu_{U(t)}$, is estimated by utilizing the geometric average of the average excess pore water pressure, $\bar{u}$, as follows:

$\mu_{U(t)}=1-\exp \left[\frac{1}{n_{\text {sim }}} \sum_{i=1}^{n_{\text {sim }}} \ln \left(\frac{\bar{u}}{u_{0}}\right)_{i}\right]$

The standard deviation of the degree of consolidation at any time $t, \sigma_{U(t)}$, is estimated as follows:

$$
\sigma_{U(t)}=\sqrt{\frac{1}{n_{s i m}-1} \sum_{i=1}^{n_{s i n}}\left[U_{i}(t)-\mu_{U(t)}\right]^{2}}
$$

where: $n_{\text {sim }}$ is the number of Monte Carlo simulations; $\left(\bar{u} / u_{0}\right)_{i}$ and $U_{i}(t)\left[=1-\left(\bar{u} / u_{0}\right)_{i}\right]$ are, respectively, the ratio of the average excess pore pressure to the initial (uniform) excess 
pore pressure and the degree of consolidation at any time $t$ for the $i$ th simulation. It has to be emphasized that, the average excess pore pressure $(\bar{u})$ is obtained by performing numerical integration over the entire area of the discretized mesh.

\section{RESULTS AND DISCUSSION}

The comparison between the axisymmetric and equivalent plane strain analyses in terms of the estimated mean and standard deviations are shown in Figures 4 and 5, in which $\mu_{U}$ and $\sigma_{U}$ are expressed as functions of the consolidation time, $t$. Since the general trend of the distribution parameters remain unaltered over the range of the statistical parameters $\omega$ and $\Theta$, the results of only a few tests are presented for brevity. In addition, it was believed that the presented tests are sufficient to demonstrate the main features of the validity assessment of the matching procedure.

Figure 4(a) shows the relationship between $\mu_{U}$ and the consolidation time, $t$, for various $\omega$ with a constant $\Theta=0.5$. It can be seen that, at any certain time $t$, the estimated values of $\mu_{U}$ derived from the equivalent plane strain analysis are slightly lower than those obtained from the axisymmetric solution, for all cases of $\omega$. The influence of $\Theta$ on $\mu_{U}$ is illustrated in Figure 4(b) for a constant $\omega=200 \%$. It can be seen that, in general, the effect of $\Theta$ on $\mu_{U}$ is marginal from the matching point of view and both solutions give almost identical results. However, at any certain consolidation time $t$, the plane strain solution gives slightly lower values of $\mu_{U}$ compared to the axisymmetric analysis, for $\Theta$ $\leq 1.0$.

The agreement between $\sigma_{U}$ estimated via axisymmetric and equivalent plane strain analyses for various values of $\omega$ at a fixed value of $\Theta=1.0$ is demonstrated in Figure 
5(a). It can be seen that the matching procedure gives identical results for both axisymmetric and plane strain when $\omega$ is as low as $25 \%$. As $\omega$ increases, the results show slight difference in $\sigma_{U}$ between the axisymmetric and plane strain especially at and near the peak value of $\sigma_{U}$. The effects of $\Theta$ on $\sigma_{U}$ is depicted in Figure 5(b) for $\omega=$ $100 \%$. General speaking, the matching procedure gives $\sigma_{U}$ curves that are exceptionally identical for axisymmetric and plane strain solutions, especially at $\Theta<1.0$. Little discrepancy, however, is observed between the axisymmetric and plane stain solutions when $\Theta \geq 1.0$.

\section{PROBABILISTIC INTERPRETATION}

One of the main objectives of the stochastic consolidation analyses is to estimate the probability that a deterministic degree of consolidation overestimates the true consolidation value. Therefore, the matching procedure between the axisymmetric and plane strain analyses is also investigated in terms of the probability of achieving a target degree of consolidation. At any given time, this involves a selection of a reasonable probability distribution for the degree of consolidation data obtained. In order to do so, the degree of consolidation data are transformed into $U^{*}(t)$, which is an alternative representing form to the degree of consolidation $U(t)$. Recalling that only the degree of consolidation due to horizontal drainage is considered in the current study, it can be assumed that $U_{h}(t)=U(t)$ and $k_{h}=k$ (as no drainage is permitted in the vertical direction). The value of $U^{*}(t)$ can then be obtained by rearranging and rewriting Eq. (1) to yield: 


$$
\ln \left[\frac{1}{1-U(t)}\right]=\frac{2 t}{R_{e}^{2} \alpha m_{v} \gamma_{w}} k
$$

Assuming that $\ln \left[1 /(1-U(t)]=U^{*}(t)\right.$, Eq. (12) becomes:

$$
U *(t)=\frac{2 t}{R_{e}^{2} \alpha m_{v} \gamma_{w}} k
$$

As indicated earlier, for an individual realization of a permeability field, $U(t)$ at each consolidation stage is computed based on the average excess pore water pressure over a large number of nodes. Therefore, the obtained $U^{*}(t)$ in Eq. (13) can be considered as the output of a single averaged permeability over this realization. If the average permeability over that realization is denoted by $\bar{k}$, Eq. (13) can then be rewritten as follows:

$U *(t)=\frac{2 t}{R_{e}^{2} \alpha m_{v} \gamma_{w}} \bar{k}$

Referring to the element permeability of Eq. (4), $\bar{k}$ can be approximated as follows:

$$
\bar{k}=\exp \left(\mu_{\ln k}+\sigma_{\ln k} \bar{g}_{\ln k}\right)
$$

where: $\bar{g}_{\ln k}$ is the arithmetic average of $g_{\ln k}$ over an individual realization of a permeability field and is normally distributed (as $g_{\ln k}$ is normally distributed). 
Therefore, $\bar{k}$ in Eq. (15) is the geometric average over that realization. Since the geometric average of a random variable tends to have a lognormal distribution by the central limit theorem, thus, $\bar{k}$ will be approximately lognormally distributed (over the suite of Monte Carlo simulation). Accordingly, it is reasonable to assume that $U^{*}(t)$ in Eq. (14) is also lognormally distributed. Based on the above hypothesis, the stochastic simulation data of $U(t)$ for each of the 60 stochastic simulation tests performed in the current study are transformed into $U^{*}(t)$ and their histograms are plotted. Figure 6 illustrates typical histograms for $U^{*}(t)$ obtained under both the axisymmetric and plane strain conditions for $\omega=100 \%$ and $\Theta=0.5$ at deterministic $90 \%$ consolidation time, $t_{\mathrm{D} 90}$, along with the fitted lognormal distributions. The goodness-of-fit test yielded $p$ values of 0.58 and 0.28 for axisymmetric and plane strain conditions, respectively, indicating good agreement between the histograms and fitted distributions. Following this process, the rationality of the lognormal distribution assumption for $U^{*}(t)$ (both for axisymmetric and plane strain analyses) is assessed through the well-known chi-square test over the entire considered range of permeability variances and scales of fluctuation at several different consolidation time. The goodness-of-fitness $p$-value never fall below 0.1 , implying that the lognormal distribution is certainly an appropriate approximation to the distribution of the simulated $U^{*}(t)$ data. Accepting the lognormal distribution for $U^{*}(t)$, the two parameters $\mu_{\ln U^{*}(t)}$ and $\sigma_{\ln U^{*}(t)}$ that are representing the mean and standard deviation of the underlying normally distributed $\ln U^{*}(t)$ are estimated for each combination of $\omega$ and $\Theta$ from the suite of 1000 realizations. For $90 \%$ target degree of consolidation, $U_{90}$ (i.e., when $\left.U=0.9\right), U^{*}=\ln [1 /(1-0.9)]=2.3026$. Therefore, the probability of getting $U^{*}(t) \geq 2.3026$ (i.e., $P\left[U^{*}(t) \geq 2.3026\right]$ ) will be equal to the 
probability of achieving $U(t) \geq 90 \%$ (i.e., $P\left[U^{*}(t) \geq U_{90}\right]$ ) and the $P\left[U(t) \geq U_{90}\right]$ can be estimated as follows:

$$
P\left[U(t) \geq U_{90}\right]=P[U *(t) \geq 2.3026]=1-\Phi\left(\frac{\ln 2.3026-\mu_{\ln U^{*}(t)}}{\sigma_{\ln U^{*}(t)}}\right)
$$

where: $P[]=$. probability of its argument and $\Phi($.$) is the standard normal cumulative$ distribution function.

Referring to the specific case shown in Figure 6, the estimated $P\left[U \geq U_{90}\right]$ for the axisymmetric case (i.e. Figure 6a) and plane strain case (i.e. Figure 6b) are $26 \%$ and $22 \%$, respectively, indicating that for the same spatial variability parameter and at a certain consolidation time, the equivalent plane strain analysis gives slightly lower probability of achieving a target degree of consolidation than the axisymmetric analysis. By making use of the estimated $\mu_{\ln U^{*}(t)}$ and $\sigma_{\ln U^{*}(t)}$ in Eq. (16), the variation of $P[U \geq$ $\left.U_{90}\right]$ with respect to the consolidation time, $t$, are obtained and the results are shown in Figure 7. Figure 7(a) shows the variation of $P\left[U \geq U_{90}\right]$ with the consolidation time for various $\omega$ at a fixed value of $\Theta=0.25$. It can be seen that the equivalent plane strain analysis gives lower values of $P\left[U \geq U_{90}\right]$ compared to the axisymmetric solution, for all cases of $\omega$. However, it can also be seen that the lower values of $P\left[U \geq U_{90}\right]$ produced by the plane strain analysis becomes more prevailing as $\omega$ increases to $400 \%$.

In Figure 7(b), the variation of $P\left[U \geq U_{90}\right]$ with the consolidation time is illustrated for various $\Theta$ at $\omega=100 \%$. It can be observed that, in general, the equivalent plane strain analysis gives slightly lower values of $P\left[U \geq U_{90}\right]$ than the axisymmetric solution, for 
all $\Theta$. However, the discrepancy between the plane strain and axisymmetric solutions become more significant for small $\Theta$. One particular note in Figure 7(b) is that, for large $\Theta>1.0$, both axisymmetric and equivalent plane strain analyses give almost identical $P\left[U \geq U_{90}\right]$, implying that the estimated $P\left[U \geq U_{90}\right]$ from both conditions will be similar if the soil is homogeneous, and vice versa. The overall observation that can be derived from comparing the results of Figures $7(a)$ and (b) is that, for erratic soils (i.e., $\Theta$ is small and $\omega$ is large), the equivalent plane strain analysis gives lower $P\left[U \geq U_{90}\right]$ than that of the axisymmetric solution.

\section{CONCLUSIONS}

The validity of the permeability matching technique that transforms the actual axisymmetric conditions to an equivalent plane strain model is examined for a reliability based analysis of soil consolidation by PVDs considering associated uncertainty due to spatial variation of soil permeability. The comparison between the plane strain and axisymmetric analyses is assessed in terms of the statistical distribution parameters and probability of achieving a target degree of consolidation. It is concluded that the equivalent plane strain analysis will always provide lower values of the mean and standard deviation of the degree of consolidation and lower probability of achieving a target degree of consolidation than the axisymmetric solution, no matter what the values of the statistical parameters are. Satisfactory matching in terms of the probability of achieving a target degree of consolidation can only be obtained when the consolidated

soil mass is more or less homogeneous (i.e., scale of fluctuation is large). For very 
erratic soils (i.e., small scale of fluctuation), the derived probability from the plane strain analysis is expected to be relatively lower than that of the axisymmetric solution.

\section{REFERENCES}

Bari, M. W., Shahin, M. A., and Nikraz, H. R. (2010). "Effects of spatial variability on consolidation of soft soil stabilized by prefabricated vertical drains." Proceedings of the 17th Southeast Asian Geotechnical Conference, Taipei, Taiwan, 43-46.

Barron, R. A. (1948). "Consolidation of fine-grained soils by drain wells." Transactions of the American Society of Civil Engineering, 113, 718-724.

Biot, M. A. (1941). "General theory of three-dimensional consolidation." Journal of Applied Physics, 12, 155-164.

Bo, M. W., Chu, J., Low, B. K., and Choa, V. (2003). Soil Improvement: prefabricated vertical drain techniques, Thomson Learning, Singapore.

Carter, J. P., and Balaam, N. P. (1995). Program AFENA - A general finite element algorithm: Users' Manual, Centre for Geotechnical Research, University of Sydney.

Fenton, A., and Vanmarcke, E. H. (1990). "Simulation of random fields via local average subdivision." Journal of Engineering Mechanics, 116(8), 1733-1749.

Fenton, G. A., and Griffiths, D. V. (2008). Risk assessment in geotechnical engineering, John Wiley and Sons, New Jersey.

Hansbo, S. (1981). "Consolidation of fine-grained soils by prefabricated drains." Proceedings of the 10th International Conference on Soil Mechanics and Foundation Engineering, Stockholm, Sweden, 677-682. 
Hird, C. C., Pyrah, I. C., and Russel, D (1992). "Finite element modelling of vertical drains beneath embankments on soft ground." Geotechnique, 42(3), 499-511.

Hoeksema, R. J., and Kitanidis, P. K. (1985). "Analysis of the spatial structure of properties of selected aquifers." Water Resources Research 21(4), 563-572.

Huang, J. , Griffiths, D. V., and Fenton, G. A. (2010). "Probabilistic analysis of coupled soil consolidation." Journal of Geotechnical and Geoenvironmental Engineering, 136(3), 417-430.

Hwang, D., and Witczak, M. W. (1984). "Multidimensional probabilistic consolidation." Journal of Geotechnical Engineering, 110(8), 1059-1077.

Indraratna, B., Bamunawita, C., Redana, I. W., and McIntosh, G. (2003). "Modelling of prefabricated vertical drains in soft clay and evaluation of their effectiveness in practice." Ground Improvement, 7(3), 127-137.

Indraratna, B., and Redana, I. W. (1997). "Plane-strain modeling of smear effects associated with vertical drains." Journal of Geotechnical and Geoenvironmental Engineering, 123(5), 474-478.

Lee, P. K., Xie, K. H., and Cheung, Y. K. (1992). "A study on one-dimensional consolidation of layered systems." International Journal of Numerical and Analytical Methods in Geomechanics, 16(11), 815-831.

Paice, G. M., Griffiths, D. V., and Fenton, G. A. (1994). "Influence of spatially random soil stiffness on foundation settlements." ASCE Geotechnical Special Publication, 1(40), 628-639.

Pyrah, I. C. (1996). "One-dimensional consolidation of layered soils." Geotechnique, $46(3), 555-560$. 
Vanmarcke, E. H. (1984). Random fields: analysis and synthesis, The MIT Press, Massachusetts. 


\section{Figure Captions:}

Figure 1. Schematic diagram of half unit cell with a prefabricated vertical drain

Figure 2. Comparison of finite element and analytical results for consolidation of a unit cell (Modified after Hird et al. 1992)

Figure 3. Typical realization of a random permeability field

Figure 4. Matching comparison for the effect of: (a) $\omega$ on $\mu_{U}$ at $\Theta=0.5$; (b) $\Theta$ on $\mu_{U}$ at $\omega=200 \%$

Figure 5. Matching comparison for the effect of: (a) $\omega$ on $\sigma_{U}$ at $\Theta=1.0$; (b) $\Theta$ on $\sigma_{U}$ at $\omega=100 \%$

Figure 6. Histogram of simulated $U^{*}$ with fitted lognormal distribution for $\omega=100 \%$, $\Theta=0.5$ at deterministic $90 \%$ consolidation time $\left(t_{\mathrm{D} 90}\right)$ (a) axisymmetric and (b) plane strain

Figure 7. Matching comparison of the effect of: (a) $\omega$ on $P\left[U \geq U_{90}\right]$ at $\Theta=0.25$; (b) $\Theta$ on $P\left[U \geq U_{90}\right]$ at $\omega=100 \%$ 


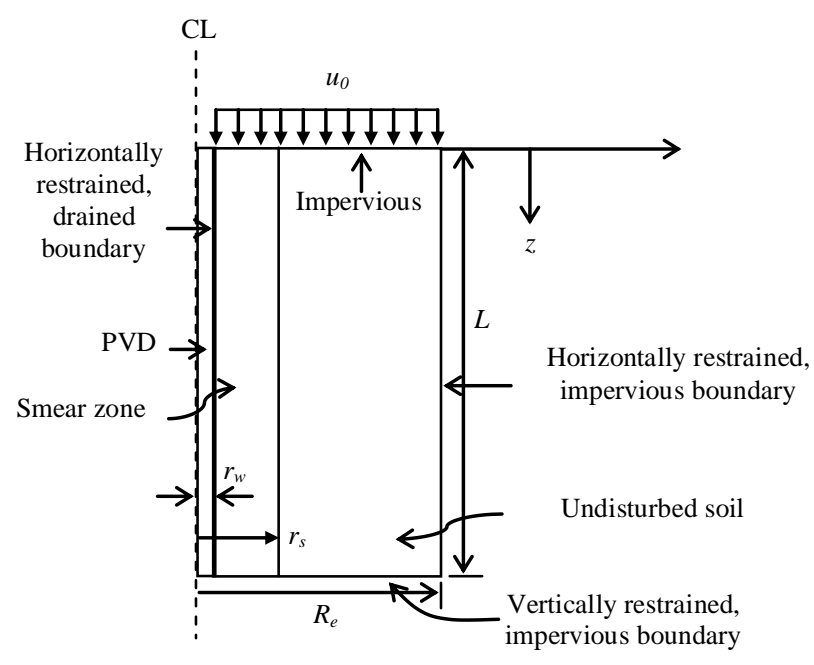

Figure 1. Schematic diagram of half unit cell with a prefabricated vertical drain 


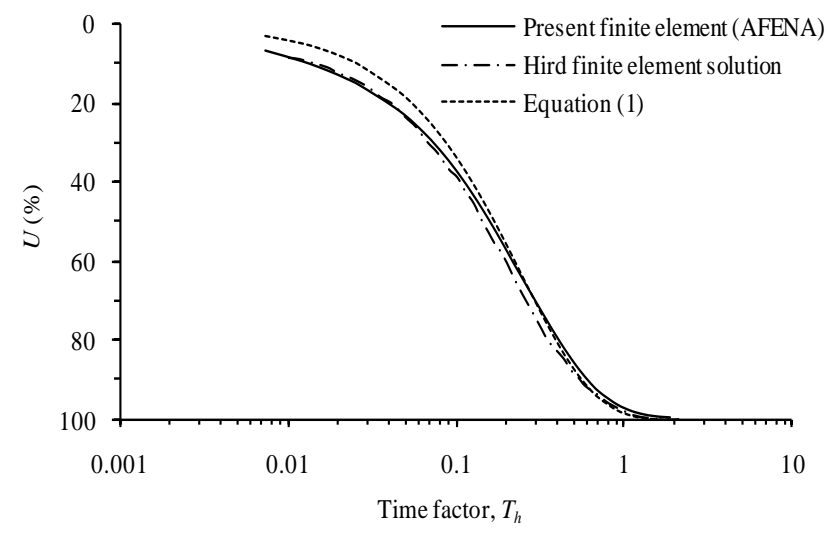

Figure 2. Comparison of finite element and analytical results for consolidation of a unit cell (Modified after Hird et al. 1992) 


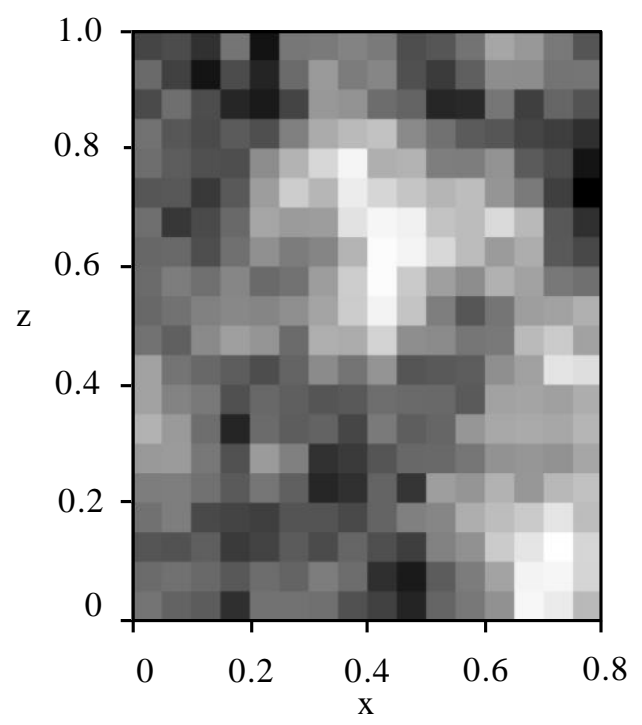

Figure 3. Typical realization of a random permeability field 

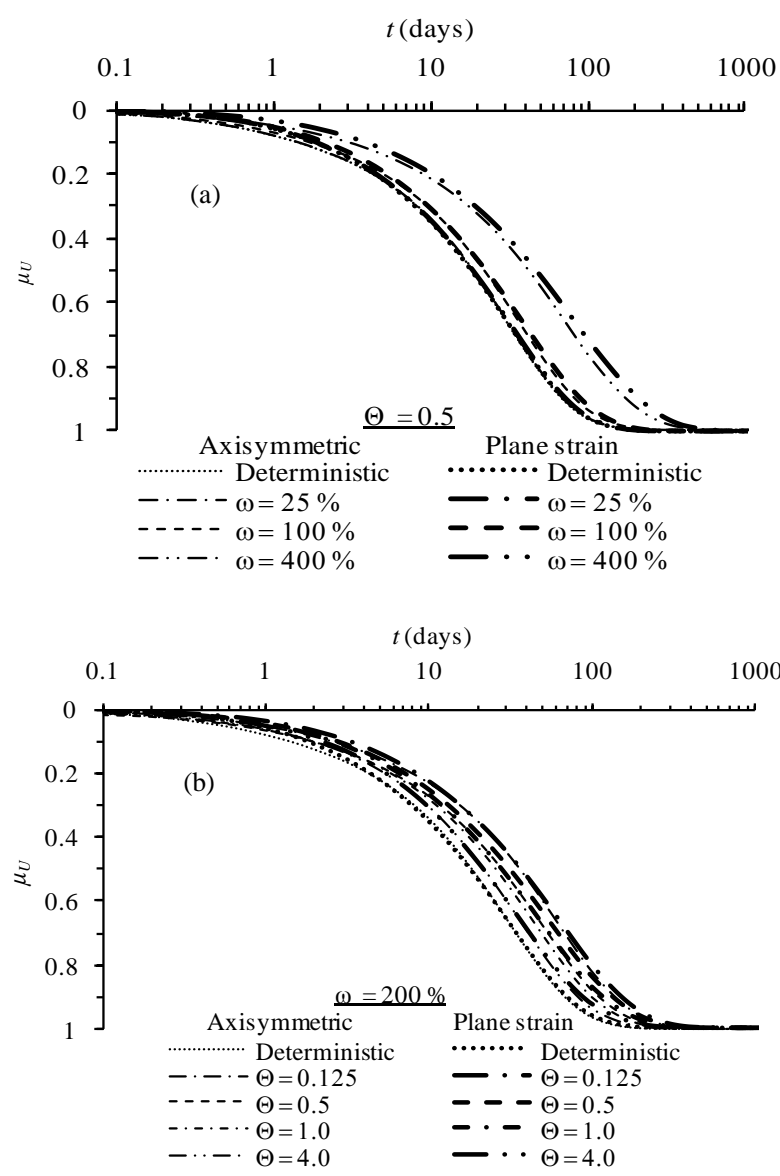

Figure 4. Matching comparison for the effect of: (a) $\omega$ on $\mu_{U}$ at $\Theta=0.5$; (b) $\Theta$ on $\mu_{U}$ at $\omega=200 \%$ 

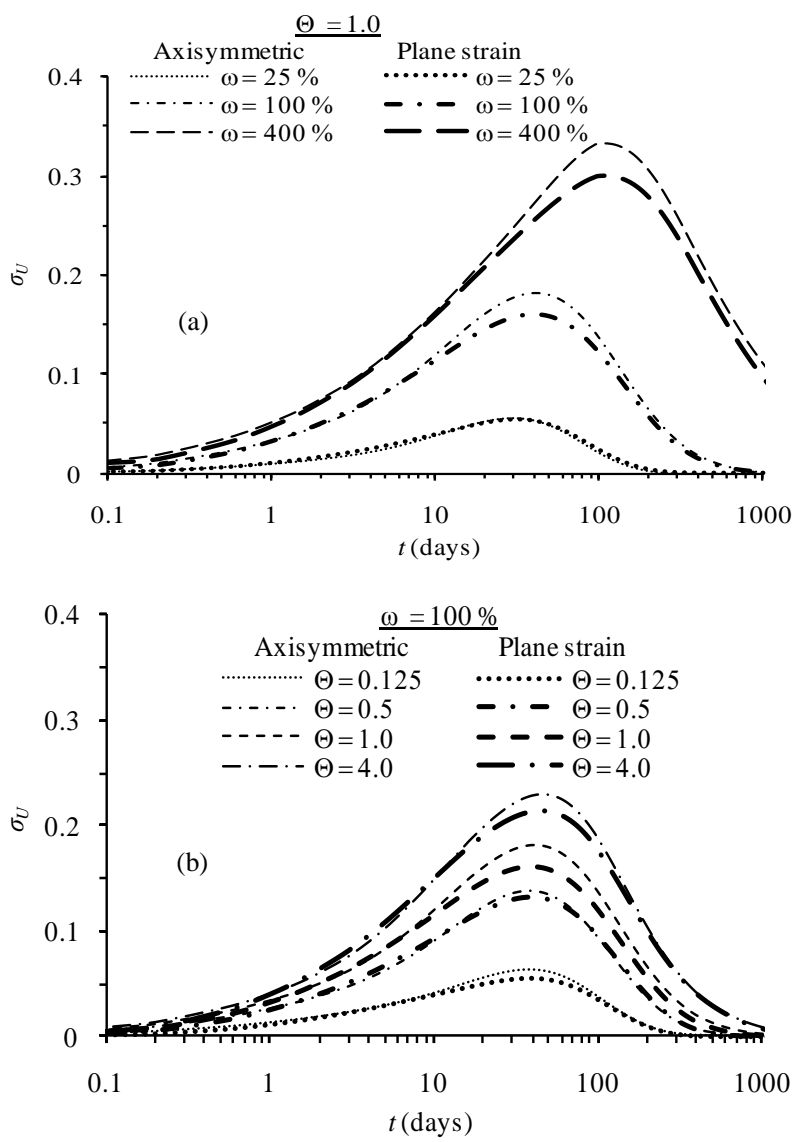

Figure 5. Matching comparison for the effect of: (a) $\omega$ on $\sigma_{U}$ at $\Theta=1.0$; (b) $\Theta$ on $\sigma_{U}$ at $\omega=100 \%$ 

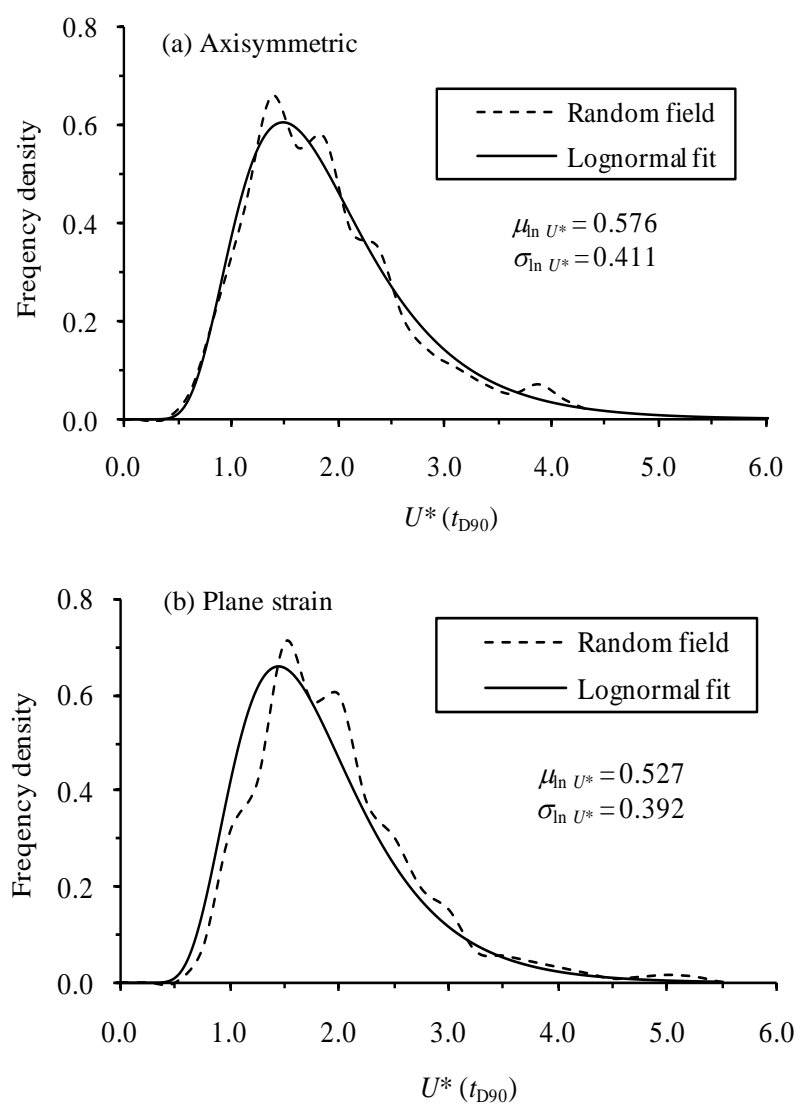

Figure 6. Histogram of simulated $U^{*}$ with fitted lognormal distribution for $\omega=100 \%$, $\Theta=0.5$ at deterministic $90 \%$ consolidation time ( $\left.t_{\mathrm{D} 90}\right)$ : (a) axisymmetric; (b) plane strain 

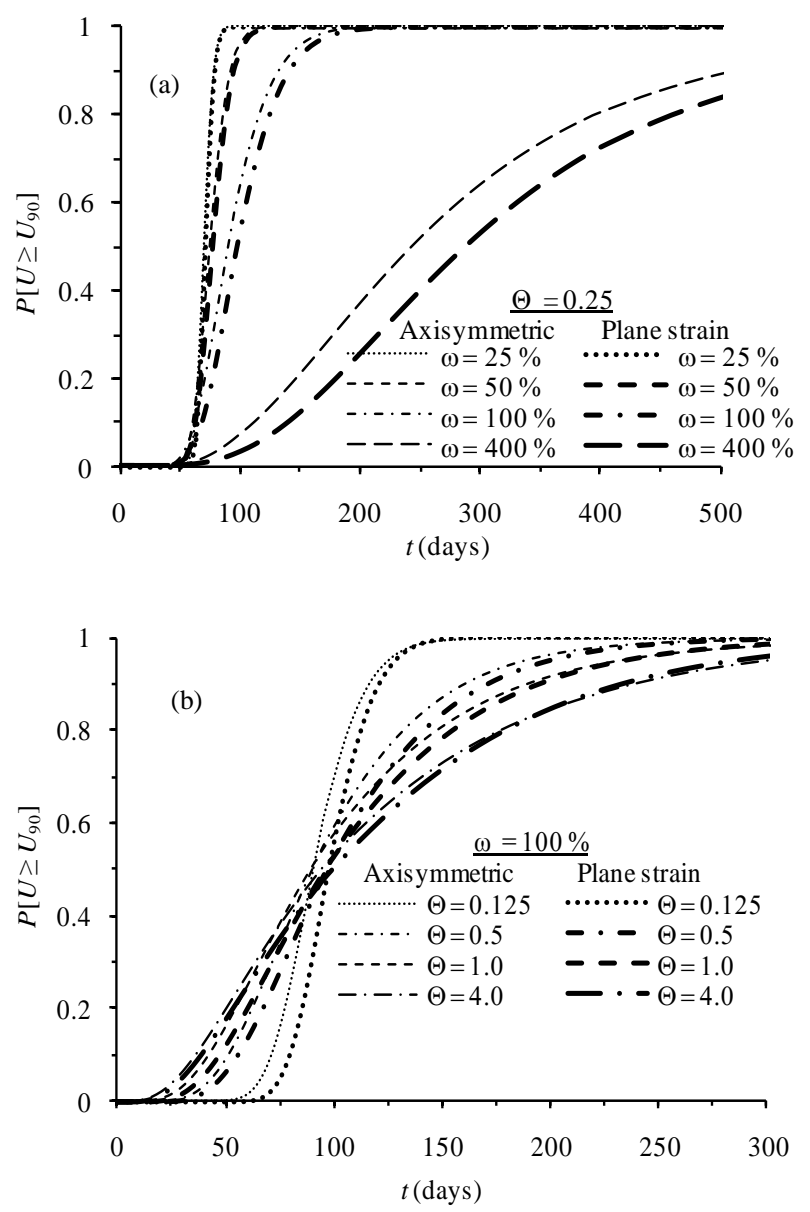

Figure 7. Matching comparison of the effect of: (a) $\omega$ on $P\left[U \geq U_{90}\right]$ at $\Theta=0.25$; (b) $\Theta$ on $P\left[U \geq U_{90}\right]$ at $\omega=100 \%$ 